\title{
Novel Findings in Obstetric Brachial Plexus Palsy: A Study of Corpus Callosum Volumetry and Resting-State Functional Magnetic Resonance Imaging of Sensorimotor Network
}

\author{
Kishore Kislay, MCh* \\ Bhagavatula Indira Devi, MS, \\ MCh* \\ Dhananjaya Ishwar Bhat, \\ MCh* \\ Dhaval Prem Shukla, MCh* \\ Arun Kumar Gupta, MD \\ Rajanikant Panda, BE \\ *Departments of Neurosurgery, Nation- \\ al Institute of Mental Health and Neu- \\ rosciences (NIMHANS), Bangalore, India; \\ ${ }^{\ddagger}$ Departments of Neuroimaging and In- \\ terventional Radiology, National Institute \\ of Mental Health and Neurosciences \\ (NIMHANS), Bangalore, India
}

The results of this study were presented (platform presentation) in the "resident awards session" at the national annual conference of Indian Society for Pediatric Neurosurgery held at Mumbai, India from March 24-26, 2017. This presentation was adjudged the best paper. The abstract/paper has not been published.

Correspondence:

Bhagavatula Indira Devi, MS, MCh,

Department of Neurosurgery,

Second floor, Neurosciences Faculty

Block,

National Institute of Mental Health and

Neurosciences (NIMHANS),

Hosur Road,

Bangalore 560029,

Karnataka, India.

E-mail: bidevidr@gmail.com

Received, April 25, 2017.

Accepted, September 11, 2017.

Published Online, October 28, 2017.

Copyright (c) 2017 by the

Congress of Neurological Surgeons
BACKGROUND: The response of the brain to obstetric brachial plexus palsy (OBPP) is not clearly understood. We propose that even a peripheral insult at the developmental stage may result in changes in the volume of white matter of the brain, which we studied using corpus callosum volumetry and resting-state functional magnetic resonance imaging (rsfMRI) of sensorimotor network.

OBJECTIVE: To study the central neural effects in OBPP.

METHODS: We performed an MRI study on a cohort of 14 children who had OBPP and 14 healthy controls. The mean age of the test subjects was $10.07 \pm 1.22 \mathrm{yr}$ (95\% confidence interval). Corpus callosum volumetry was compared with that of age-matched healthy subjects. Hofer and Frahm segmentation was used. Resting-state fMRI data were analyzed using the FSL software (FMRIB Software Library v5.0, Oxford, United Kingdom), and group analysis of the sensorimotor network was performed.

RESULTS: Statistical analysis of corpus callosum volume revealed significant differences between the OBPP cohort and healthy controls, especially in the motor association areas. Independent t-test revealed statistically significant volume loss in segments I (prefrontal), II (premotor), and IV (primary sensory area). rsfMRI of sensorimotor network showed decreased activation in the test hemisphere (the side contralateral to the injured brachial plexus) and also decreased activation in the ipsilateral hemisphere, when compared with healthy controls.

CONCLUSION: OBPP occurs in an immature brain and causes central cortical changes. There is secondary corpus callosum atrophy which may be due to retrograde transneuronal degeneration. This in turn may result in disruption of interhemispheric coactivation and consequent reduction in activation of sensorimotor network even in the ipsilateral hemisphere.

KEY WORDS: Obstetric brachial plexus palsy, Resting-state fMRI, Cortical plasticity, Corpus callosum volume

Neurosurgery 83:905-914, 2018

DOI:10.1093/neuros/nyx495

www.neurosurgery-online.com

$\mathbf{T}$

he human brain continues to develop well beyond the fetal period as the process of myelination and maturation occurs. ${ }^{1-3}$

Maturation involves formation of new synapses. Obstetric brachial plexus palsy (OBBP) occurs at the time of childbirth, when none of these are

complete. The brain's response to OBBP is not well established.

Brachial plexus injury (BPI) results in insult to the descending motor projections and sensory input. Alterations in the circuitry of brain may be expected to occur in OBPP, which occurs at an immature stage of brain development.

ABBREVIATIONS: BPI, brachial plexus injury; DTI, diffusion tensor imaging; EPI, echo-planar images; FSL, FMRIB Software Library; GLM, general linear model; IC, independent component; ICA, independent component analysis; MELODIC, multivariate exploratory linear decomposition into independent components; OBPP, obstetric brachial plexus palsy; PICA, probabilistic independent component analysis; rsfMRI, resting-state functional magnetic resonance imaging; TFCE, threshold-free cluster enhancement 


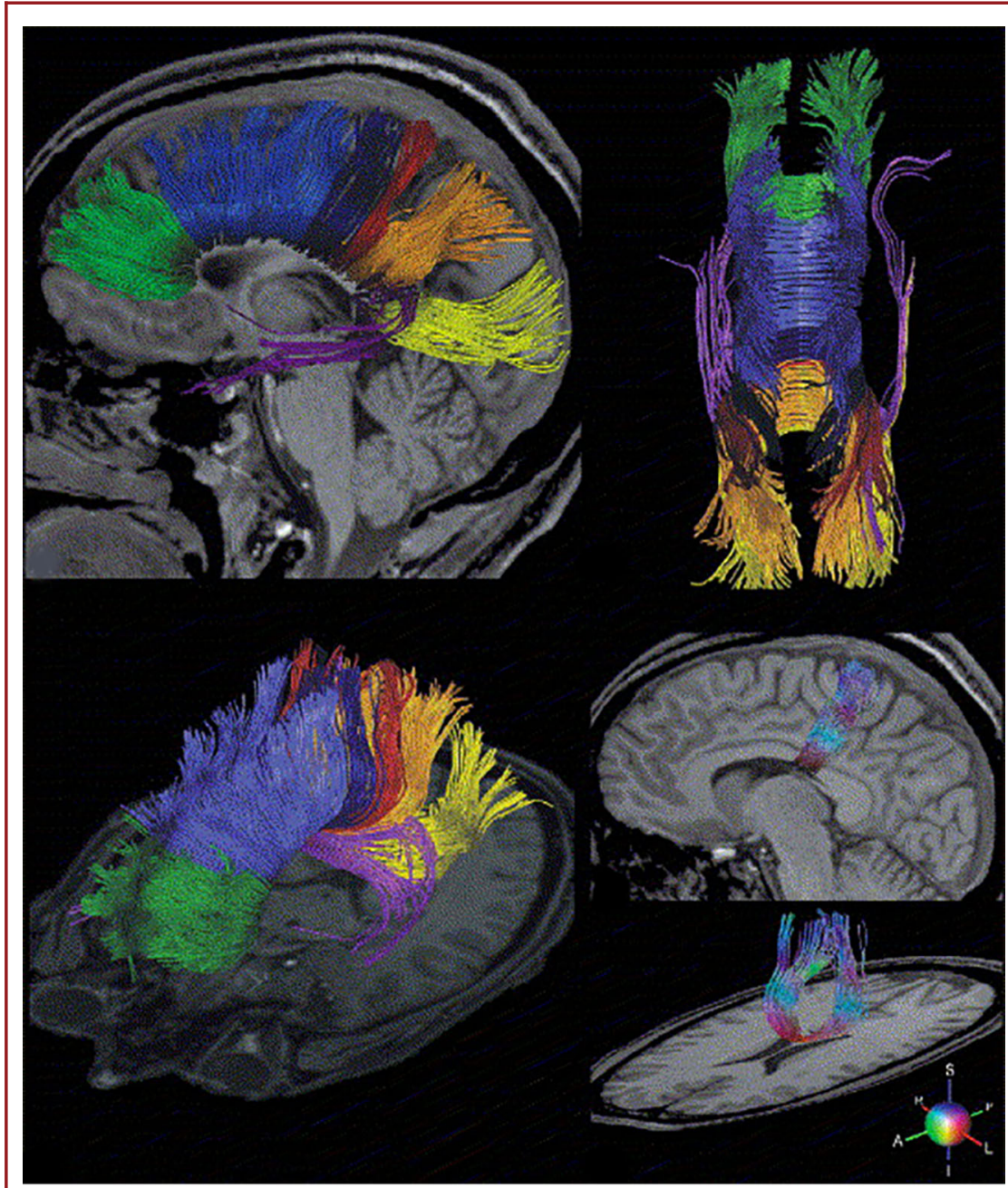

FIGURE 1. Transcallosal fiber tracts from a single male subject overlaid onto individual anatomical reference images. Sagittal, top, and oblique views of a 3-dimensional reconstruction of all callosal fibers comprising bundles projecting into the prefrontal lobe (coded in green), premotor and supplementary motor areas (light blue), primary motor cortex (dark blue), primary sensory cortex (red), parietal lobe (orange), occipital lobe (yellow), and temporal lobe (violet). Sagittal and oblique views of callosal fiber tracts that project into the primary motor cortex. The colors correspond to the local mean diffusion direction as indicated by the color code in the lower right of the figure: $A$, anterior; I, inferior; $L$, left; $P$, posterior; $R$, right; $S$, superior. Reprinted from Hofer and Frahm, ${ }^{23}$ with permission from Elsevier.

Following peripheral nerve injury, there is anterograde (Wallerian) and retrograde axonal degeneration. Due to loss of afferent and efferent signals and disruption of cortical input, cortical inactivity and degeneration is only to be expected. . $^{4-6}$ This in turn may result in changes in white matter volume and interhemispheric connectivity. ${ }^{7-10}$ White matter volume changes would be reflected in corpus callosum volumetry. ${ }^{11-15}$ As a corollary, disruption of interhemispheric coactivation may result in bilateral effects on the brain.

Resting-state functional magnetic resonance imaging (rsfMRI) of the brain is a technique wherein multiple functional networks can be studied in a brain. ${ }^{16-18}$ This is especially helpful in subjects who are unable to perform the required task. Multiple studies have been performed in adults with traumatic BPI using rsfMRI, and cortical plasticity has been demonstrated. ${ }^{19-22}$ It is a good tool to study the pattern of interhemispheric coactivation.

The corpus callosum is the main white matter bundle which connects both the hemispheres and is the major substrate for interhemispheric coactivation. Interthalamic adhesion and anterior commissure may have minor contributions. ${ }^{7,9,10}$ Diffusion tensor imaging and fiber tractography have shown that different segments of corpus callosum carry fibers from 


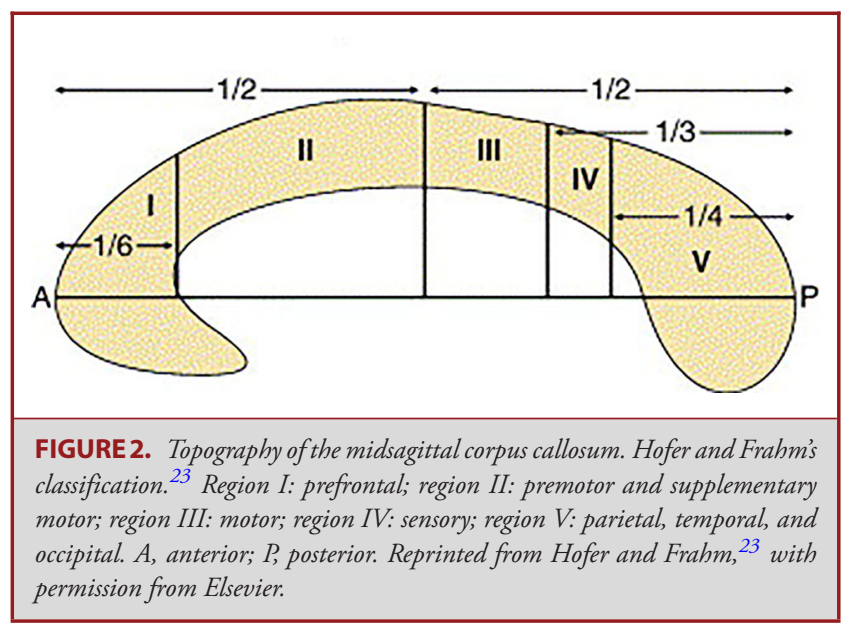

different regions of the cerebrum ${ }^{23}$ (see Figures 1 and 2). Hofer and $\mathrm{Frahm}^{23}$ parcellated the corpus callosum into 5 segments based on the cerebral regions:

- Segment I-anterior sixth; prefrontal area

- Segment II-beyond anterior sixth up to anterior half; premotor and supplementary motor area

- Segment III—first sixth beyond anterior half; primary motor area

- Segment IV—first twelfth beyond segment III; primary sensory area

- Segment V—posterior quarter; parietal-temporal-occipital area

\section{Objectives}

This study was aimed to examine the central neural effects of OBPP, which is a peripheral nerve injury, in a developing brain.

\section{METHODS}

\section{Study Design}

This was an observational cross-sectional study conducted over 3 years (2013-2016). Patients were recruited from neurosurgery outpatient department of our hospital.

\section{OBPP Group}

The inclusion criterion was occurrence of OBPP, irrespective of previous intervention. Following institute ethics committee clearance, 14 patients were included in the study. The mean age of the subjects was $10.07 \pm 1.22 \mathrm{yr}(95 \%$ confidence interval). Children with history of perinatal asphyxia (determined by either documentation in obstetrical records or MRI features suggestive of encephalomalacia/leukomalacia or deep nuclei changes) or hydrocephalus, which could confound volumetric analysis, were excluded from the study.

\section{Healthy Controls}

Fourteen age-matched healthy controls were taken from our preexisting database. Mean age was $12.92 \pm 1.50$ yr $(95 \%$ confidence interval).

\section{Ethical Clearance}

The consent for participating in the study was obtained from the child's parents and also the child. They were explained that regardless of the inclusion, standard care would be continued. A clinical proforma was generated and all the demographic details were collected.

\section{MRI Data Acquisition}

Resting-state fMRI was acquired using a 3T scanner (Skyra, Siemens, Erlangen, Germany). We use these MRI parameters for all the rsfMRI-based studies in our Institute. One hundred eighty-five volumes of gradient-echo, echo-planar images (EPI) were obtained using the following parameters: 36 slices, $4 \mathrm{~mm}$ slice thickness in interleaved manner with a field of view of $192 \times 192 \mathrm{~mm}$, echo time $35 \mathrm{~ms}$, repetition time $3000 \mathrm{~ms}$, re-focusing pulse $90^{\circ}$, voxel size- $3 \times 3 \times 4 \mathrm{~mm}$, and matrix- $256 \times 256 \times 114$. A T1-weighted 3-dimensional magnetization prepared rapid acquisition gradient echo (T1 3D MPRAGE) sequence was acquired for anatomical information (with the voxel size $1 \times 1 \times 1 \mathrm{~mm}, 192 \times 192 \times 256$ matrix).

\section{Corpus Callosum Volumetry}

Corpus callosum volumetry was done using a rule-based automated segmentation technique with the help of C8 software, ${ }^{24}$ which is based on SPM platform.

\section{Workflow ${ }^{25}$}

1. Data preparation

2. Preprocessing

a. Midsagittal plane extraction

b. Automated corpus callosum segmentation with optional manual editing

c. Thickness profile generation and areal parcellation

d. Project segmentation and parcellations to native space

3. Statistical testing of group-wise thickness profile.

We used Hofer and Frahm ${ }^{23}$ parcellation technique for segmental volumetry.

\section{Resting-State fMRI Preprocessing}

Analysis of rsfMRI images was done using FSL (FMRIB Software Library v5.0, Oxford, United Kingdom). ${ }^{26}$ Independent component analysis (ICA) was performed, and sensorimotor network was identified. The main tools used were fMRI Expert Analysis Tool and multivariate exploratory linear decomposition into independent components (MELODIC) modules. Signal equilibration was done after discarding first 5 functional images (EPI volumes) and 180 volumes were used for analysis. MCFLIRT ${ }^{27}$ was used for motion correction, and Brain Extraction Toolbox ${ }^{28}$ was used to remove nonbrain tissue (cerebrospinal fluid, scalp, etc). There were no significant head motion-related artifacts between the groups. Spatial smoothing was done using techniques described in the literature. ${ }^{26,29,30}$ EPI volumes of each individual subject's high-resolution anatomical volumes were co-registered into MNI152 standard space template, and re-sampling was performed keeping the resolution at $3 \mathrm{~mm}$ using FNIRT (FMRIB nonlinear image registration tool). ${ }^{31}$

\section{Independent Components}

The technique of ICA was used to ascertain the functional connectivity. This data-driven approach allows us to extract voxel-wise 


\begin{tabular}{|c|c|c|c|c|c|c|}
\hline & Brain region & X co-or & Y co-or & Z co-or & K (no. of voxels) & T-value \\
\hline \multirow[t]{2}{*}{ Patient_Right (internal control) } & Postcentral_R & 42 & -26 & 60 & 950 & 8.1 \\
\hline & Precentral_R & 32 & -14 & 59 & 451 & 6.2 \\
\hline \multirow[t]{2}{*}{ Patient_Left (test) } & Postcentral_L & -48 & -23 & 55 & 494 & 7.3 \\
\hline & Precentral_L & -30 & 18 & 60 & 221 & 6 \\
\hline \multirow[t]{2}{*}{ Healthy Control_Right } & Postcentral_R & 36 & -34 & 57 & 1375 & 11.9 \\
\hline & Precentral_R & 38 & -13 & 60 & 1031 & 9.2 \\
\hline \multirow[t]{2}{*}{ Healthy Control_Left } & Postcentral_L & -35 & -34 & 52 & 1606 & 12.3 \\
\hline & Precentral_L & -25 & -26 & 67 & 1476 & 9.4 \\
\hline
\end{tabular}

functional networks based on their temporal and spatial fluctuations. FSL's MELODIC tool ${ }^{29,30,32}$ was used to perform the probabilistic independent component analysis (PICA) and its data exploration was used to perform single-subject ICA. All extracted independent components (ICs) were visually inspected. Eye motion-related artifacts, highfrequency noise, field inhomogeneity, gradient instability, slice dropout, and breathing and heart rate-related artifacts were de-noised based on the existing literature using the command "fsl_regfilt" defined in FSL MELODIC. ${ }^{26,29,30}$ The same tool was also used to perform multivariate group PICA and derive spatially ICs across all datasets (14 test subjects and 14 controls) which were temporally concatenated. Fast ICA algorithm was used to optimize the non-Gaussian spatial source distributions of 70 sets of independent vectors across temporal and spatial domains.

\section{Dual Regression Analysis}

Subject-specific time series and associated spatial maps were generated from the spatial maps obtained from group ICs. ${ }^{32,33}$ The data components representing the sensorimotor area were identified from the set of group IC maps by visually comparing their anatomical location. The left and right hemisphere sensorimotor ICs are selected from the group ICs. To compare the injured hemisphere with healthy hemisphere of the control group, group-wise comparison was performed using dual regression. Statistical differences were assessed using thresholdfree cluster enhancement (TFCE) technique. ${ }^{34}$ Dual regression was carried out on the group IC data implemented in FSL, which allows voxel-wise comparisons of resting-state functional connectivity. It is a regression technique that performs reverse engineering of individual subject level data from each group-level component map (https://fsl.fmrib.ox.ac.uk/fsl/fslwiki/DualRegression). ${ }^{26}$ The set of spatial maps derived from group-mean analysis was used to generate individual subject-specific spatial maps and temporal dynamics. This approach proceeds in 2 steps: the first involves the use of a full set of group IC spatial maps in a linear spatial regression model against separate fMRI datasets, providing the matrices describing subject-specific and component-specific temporal dynamics. Next, these time-course matrices for each subject are used to perform linear temporal regression against the associated rsfMRI dataset to generate subject-specific spatial maps. Finally, different component maps are collated across the subjects into a single $4 \mathrm{D}$ file. At this stage, the ICs of interest are identified for further analysis. In this study, left and right hemispheric sensorimotor networks were the IC components of interest, which were visually identified. Group differences for each spatial map were assessed using a voxel-wise general linear model (GLM)-based analysis. Nonparametric permutation (5000 permutations)-based testing with cluster-based thresholding using TFCE with cluster significance threshold of $P<.05$ was used to obtain suprathreshold cluster map. This resulted in spatial maps characterizing the test and control group differences. The GLM comparison included (1) injured hemisphere with healthy hemisphere of patient group and (2) patient and control group. Each of the components of interest was tested for difference between groups and group averages. To estimate group mean effects, the results were thresholded with familywise error corrected at $P \leq .05$, applied for voxel-wise data. Then we used a false discovery rate correction $(P<.05)$ for multiple comparisons on top of TFCE suprathreshold maps of each group. However, we did not find statistical significance due to limited number of samples. Therefore, the group differences were quantified by the cluster values and the intensity t-value of ICs spatial maps which were extracted after TFCE threshold for each group and condition (see Table 1).

Using the paradigm of experimental vs control, in a right BPI, the left hemisphere was presumed to be the "test" side and the right would be "internal control." Subgroup analysis was performed among the 3 groups: test, internal control, and healthy control.

The results in test subjects with corresponding hemispheres of healthy controls were also compared. During the study, we were curious to see what differences were in those who underwent intervention and those who did not. However, as the ICA of rsfMRI was performed for the entire group as a whole, we could not derive any meaningful results due to the small numbers. It was not technically feasible to subdivide patients into operated and nonoperated. The final output of sensorimotor network activation in rsfMRI is a mean of a group of patients. Usually, for meaningful results (ie, summated averaging of output for all patients), numbers in excess of 10 subjects are required. In this study, the small numbers did not permit us to further subanalyze the operated and nonoperated subjects.

Since the test group was heterogenous in terms of right and left side of obstetric BPI, the images were appropriately flipped to orient all the test hemispheres on left side. All the healthy controls were right-handed individuals.

\section{Statistical Analysis}

Statistical analysis was done using SPSS (IBM Corp. Released 2011. IBM SPSS Statistics for Windows, Version 20.0. Armonk, New York).

Two-tailed $\mathrm{t}$-test was performed to detect significance $(P<.05)$ between the segmental volumetry between the 2 groups. 
TABLE 2. Clinical Profile of Patients with OBPP Who Underwent Neurotization

\begin{tabular}{|c|c|c|c|c|c|c|c|c|c|c|}
\hline Subject & $\begin{array}{c}\text { Age (in years) } \\
\text { at presentation }\end{array}$ & Side & Diagnosis & \multicolumn{3}{|c|}{$\begin{array}{c}\text { Power at } \\
\text { first visit } \\
\text { (MRC grading 0-5) }\end{array}$} & Horner's & Intervention & $\begin{array}{l}\text { Outcome at } \\
\text { 1-yr follow up } \\
\text { (MRC } \\
\text { grading 0-5) }\end{array}$ & $\begin{array}{l}\text { Narakas' } \\
\text { grade } \\
\text { (at birth) }\end{array}$ \\
\hline KT & 11 & Right & Erb's & 3 & 3 & 5 & No & $\begin{array}{l}\text { Pectoralis release } \\
\text { elsewhere at age } 3 \mathrm{yr}, \\
\text { followed by axillary-IC } \\
\text { neurotization at age } 11 \mathrm{yr}\end{array}$ & $\begin{array}{c}\text { Improved to } \\
4 / 5\end{array}$ & I \\
\hline AG & 9 & Left & Erb's & 3 & 3 & 5 & No & $\begin{array}{l}\text { IC-MC neurotization at } \\
\text { age } 9 \mathrm{yr}\end{array}$ & $\begin{array}{l}\text { Improved to } \\
4 / 5\end{array}$ & I \\
\hline NP & 8 & Right & Erb's & 3 & 3 & 5 & No & $\begin{array}{l}\text { IC-MC neurotization at } \\
\text { age } 8 \mathrm{yr}\end{array}$ & $\begin{array}{l}\text { Improved to } \\
4 / 5\end{array}$ & I \\
\hline PK & 12 & Left & Pan-plexus & 0 & 0 & 1 & yes & $\begin{array}{l}\text { IC-MC neurotization at } \\
\text { age } 5 \mathrm{yr}\end{array}$ & Not improved & IV \\
\hline
\end{tabular}

IC = intercostal nerve; $M C=$ musculocutaneous nerve; $M R C=$ Medical Research Council; Sh.abd = shoulder abduction; elb.flex $=$ elbow flexion.

TABLE 3. Clinical Profile of Patients With OBPP Who Did Not Undergo Surgical Intervention

\begin{tabular}{|c|c|c|c|c|c|c|c|c|c|c|}
\hline Subject & $\begin{array}{l}\text { Age (in years) } \\
\text { at presentation }\end{array}$ & Side & Diagnosis & \multicolumn{3}{|c|}{$\begin{array}{c}\text { Power at } \\
\text { first visit } \\
\text { (MRC grading 0-5) }\end{array}$} & Horner's & Intervention & $\begin{array}{l}\text { Outcome at } \\
\text { 1-yr follow up } \\
\text { (MRC } \\
\text { grading 0-5) }\end{array}$ & $\begin{array}{l}\text { Narakas' } \\
\text { grade } \\
\text { (at birth) }\end{array}$ \\
\hline TJ & 12 & Left & Erb's & 4 & 4 & 5 & No & $\begin{array}{l}\text { Advised } \\
\text { neurotization. } \\
\text { Parents refused }\end{array}$ & $? ?$ & 1 \\
\hline DK & 5 & Right & Pan-plexus & 3 & 3 & 3 & No & Physiotherapy & Unchanged & III \\
\hline VL & 10 & Right & Pan-plexus & 3 & 3 & 0 & yes & Physiotherapy & Unchanged & IV \\
\hline GK & 13 & Right & Pan-plexus & 3 & 3 & 0 & yes & Physiotherapy & Unchanged & IV \\
\hline YO & 11 & Right & Pan-plexus & 3 & 3 & 0 & Yes & Physiotherapy & Unchanged & IV \\
\hline SJ & 9 & Right & Pan-plexus & 1 & 1 & 1 & yes & Physiotherapy & Unchanged & IV \\
\hline HK & 10 & Right & Pan-plexus & 1 & 1 & 1 & No & Physiotherapy & Unchanged & III \\
\hline
\end{tabular}

$\mathrm{IC}=$ intercostal nerve; $\mathrm{MC}=$ musculocutaneous nerve; $\mathrm{MRC}=$ Medical Research Council; Sh.abd = shoulder abduction; elb.flex = elbow flexion.

For analysis of the results of rsfMRI, visual inspection of the images provided a subjective idea of the differential activation of the 2 sides. Further statistical quantification was made using dual regression analysis comparing the cluster values and $t$-values of the intensity of activation.

\section{RESULTS}

\section{OBPP Group}

The mean age in the current study was $10.07 \pm 1.22 \mathrm{yr}(95 \%$ confidence interval). Full-scale Medical Research Council grading and Narakas' grading ${ }^{36}$ were used for clinical classification. Five of them had Erb's palsy and rest of them had pan-plexus palsy. All patients had zero active range of motion in shoulder and elbow at birth, and had been on regular physiotherapy. Immediate preoperatively $(n=4)$, power was $3 / 5$ in 3 cases and $0 / 5$ in 1 patient. Most of them (9/14) did obtain partial improvement in motor strength by the time they were referred to us. Postneurotization $(\mathrm{n}=4)$ improvement was observed in 3 out of 4 patients. The only surgical intervention done prior to consultation with us was pectoralis release in one patient. Patients with Narakas' grade III and IV at birth did not recover well. Recovery was universally poor in the distal muscles (see Tables 2 and 3). 
TABLE 4. Segmental Corpus Callosum Volume in Subjects With OBPP (Test) and Healthy Controls

\begin{tabular}{|c|c|c|c|c|c|c|c|c|c|c|c|c|c|}
\hline \multicolumn{2}{|c|}{ Subject } & \multicolumn{2}{|c|}{ Segment I } & \multicolumn{2}{|c|}{ Segment II } & \multicolumn{2}{|c|}{ Segment III } & \multicolumn{2}{|c|}{ Segment IV } & \multicolumn{2}{|c|}{ Segment V } & \multicolumn{2}{|c|}{ Total volume } \\
\hline Test & Control & Test & Control & Test & Control & Test & Control & Test & Control & Test & Control & Test & Control \\
\hline KT & $\mathrm{HC1}$ & 52.6875 & 60.0625 & 98.125 & 93.25 & 33.3125 & 32.8125 & 19.25 & 9.9375 & 109 & 57.25 & 312.375 & 253.3125 \\
\hline$A G$ & $\mathrm{HC} 2$ & 67 & 98.3125 & 119 & 159.625 & 46.1875 & 61.25 & 15.0625 & 32.875 & 89.125 & 142.375 & 336.375 & 494.4375 \\
\hline NP & $\mathrm{HC} 3$ & 68.9375 & 69.6875 & 134.5625 & 105.6875 & 44.1875 & 39.75 & 14.125 & 17.3125 & 127.875 & 93.5625 & 389.6875 & 326 \\
\hline PK & $\mathrm{HC} 4$ & 69.3125 & 79.5625 & 121.3125 & 120.0625 & 54.875 & 30.3125 & 28.25 & 14.8125 & 155.25 & 118.1875 & 429 & 362.9375 \\
\hline TJ & HC5 & 51.625 & 56.6875 & 116.625 & 100.375 & 51.125 & 52 & 20 & 20.1875 & 118 & 100.4375 & 357.375 & 329.6875 \\
\hline DK & $\mathrm{HC} 6$ & 56.9375 & 68.4375 & 96 & 101.0625 & 51.3125 & 55.125 & 22.1875 & 22.5625 & 104 & 115.9375 & 330.4375 & 363.125 \\
\hline SK & $\mathrm{HC7}$ & 15.9375 & 105.5 & 49.25 & 157.1875 & 23.9375 & 59.9375 & 11.125 & 31.375 & 24.8125 & 136.375 & 125.0625 & 490.375 \\
\hline SJ & $\mathrm{HC} 8$ & 70.5625 & 48.5 & 109.1875 & 115.5 & 41.625 & 41.75 & 23.25 & 18.8125 & 112.4375 & 88.8125 & 357.0625 & 313.375 \\
\hline VL & $\mathrm{HC} 9$ & 47.75 & 95.8125 & 103.125 & 134.8125 & 51.1875 & 57.875 & 20.25 & 30 & 78.4375 & 140.5625 & 300.75 & 459.0625 \\
\hline GK & $\mathrm{HC} 10$ & 64.625 & 85.625 & 76.875 & 137.375 & 30.375 & 40.375 & 14.4375 & 35.75 & 73.5 & 150.5 & 259.8125 & 449.625 \\
\hline $\mathrm{HF}$ & HC11 & 51.625 & 81.3125 & 97.25 & 150.3125 & 48.375 & 66.125 & 19.5 & 31.6875 & 128.375 & 169.0625 & 396.125 & 498.5 \\
\hline HK & $\mathrm{HC} 12$ & 53.8125 & 67.1875 & 96.3125 & 94.625 & 41.725 & 53.9375 & 18.625 & 19 & 82.375 & 90.5 & 316.375 & 325.25 \\
\hline YO & $\mathrm{HC} 13$ & 60.1875 & 76.5625 & 106.3125 & 124.0625 & 38.625 & 63.875 & 16.0625 & 38.4375 & 89.0625 & 117.75 & 358.25 & 385.1875 \\
\hline SG & $\mathrm{HC} 14$ & 62.75 & 94.8125 & 110.0625 & 144.5625 & 40.875 & 68.5 & 18.0625 & 34.4375 & 97.0625 & 140.0625 & 361.8125 & 482.375 \\
\hline Total & & 793.75 & 1180.25 & 1434 & 1894.563 & 597.725 & 786.1875 & 260.1875 & 385.6125 & 1389.313 & 1834.313 & 4630.5 & 6045.425 \\
\hline
\end{tabular}

\section{Corpus Callosum Volumetry}

Statistical analysis of corpus callosum volume revealed significant differences between the OBPP cohort and healthy controls (see Table 4). The mean corpus callosum volume was lower in all segments of corpus callosum in OBPP cohort as compared to that in healthy controls.

Independent t-test revealed statistically significant volume loss in segments I, II, and IV, and narrowly missed significance mark in segment III (corresponding to fibers from primary motor region; see Figure 3/Table 5).

\section{Resting-State fMRI Results}

\section{Test Hemisphere (Left) vs Internal Control (Right)}

There is decreased activation in the sensorimotor network on the left (test) hemisphere, and is predominantly in the postcentral region. In the right hemisphere (internal control) of the patient, a total of 1401 voxels were activated in the sensorimotor network, compared to 715 voxels on the left side (test side; see Figures 4A and $4 \mathrm{~B} /$ Table 1 ).

\section{Test Hemisphere (Left) vs Corresponding (Left) Healthy Control}

There is marked reduction in activation of sensorimotor network and extent of sensorimotor network in the test hemisphere as compared to the same side in healthy controls. A nonquantitative visual assessment reveals a shrunken and weakly activated sensorimotor network in test cohort. The activated number of voxels is more than thrice as large (3082 vs 715) in healthy subjects (see Figures $4 \mathrm{~B}$ and 4D/Table 1).
Internal Control (Right) vs Corresponding (Right) Healthy Control

Although the activation is stronger on the internal control as compared to test side, it is still weaker than that in a healthy control, essentially amounting to only $60 \%$ of the healthy value (see Figures 4A and 4C/Table 1).

\section{DISCUSSION}

To the best of our knowledge, this is the first of its kind to study corpus callosum volumetry and application of rsfMRI of sensorimotor network in OBPP. It attempts to highlight the central effects of a peripheral, indirect, insult to the brain.

\section{Cortical Plasticity and Application of rsfMRI}

The sensorimotor network has been the point of interest in various studies on BPI, ${ }^{34}$ and following peripheral nerve surgeries. Substantial literature exists which have demonstrated good correlation between the results of task-based fMRI and rsfMRI. ${ }^{17,18,35-37}$

An important difference between adult plasticity and developmental plasticity (which occurs in the developing brain) is that pediatric brain has a stronger plastic potential. Various growth factors are lost at various stages in the developmental period, ${ }^{38}$ rendering plasticity more uncertain at later years.

Completion of peripheral innervation and physical integrity of the neural circuit is not equivalent to functional outcomes, although this is a mandatory first step. Unless "meaningful" cortical re-organization occurs, the "wiring" cannot be activated. This explains why some patients do well and some do not, despite a successful neurotization. ${ }^{39}$ Sokki et al ${ }^{20}$ demonstrated 


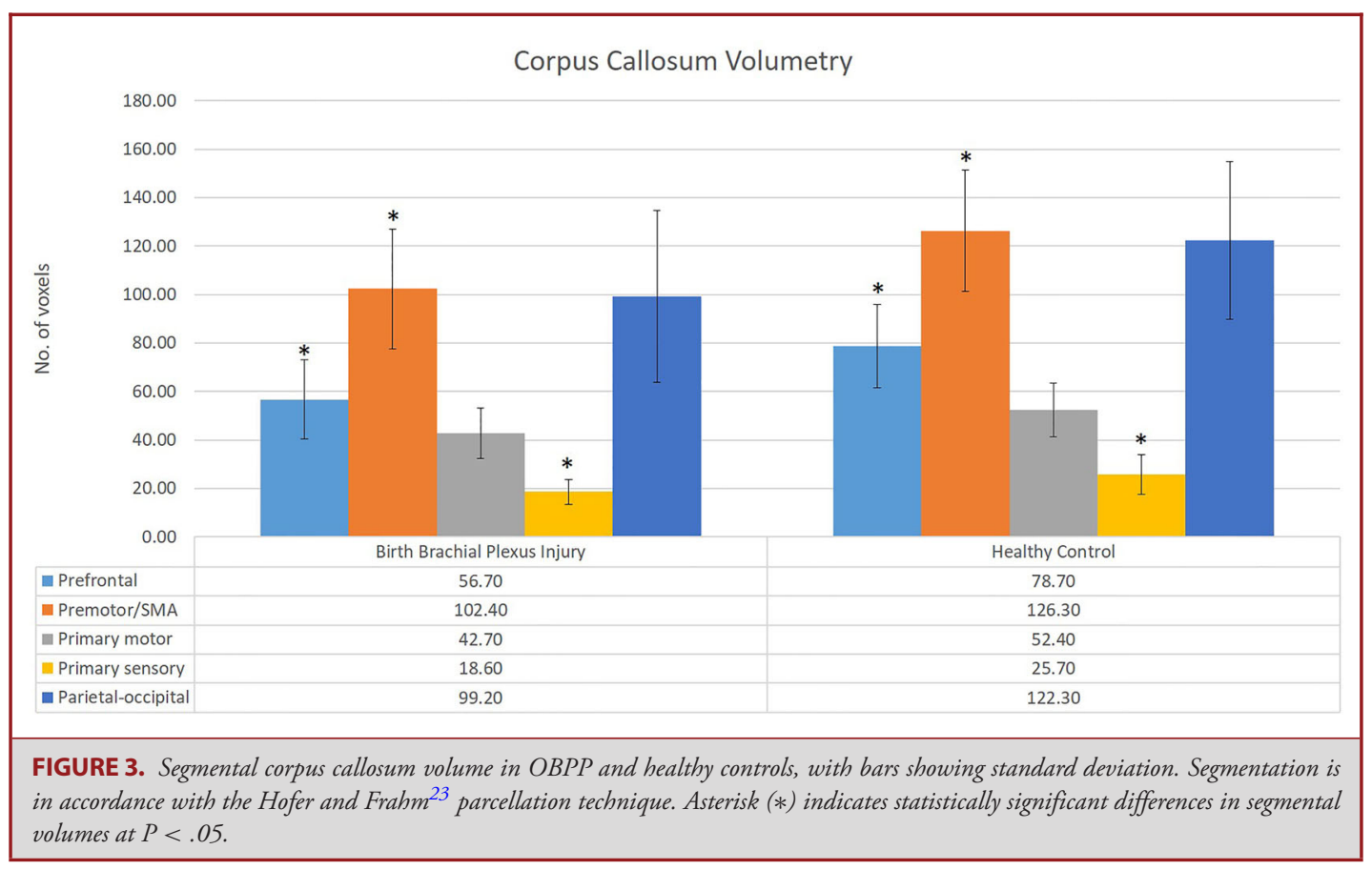

\begin{tabular}{|c|c|c|c|}
\hline $\begin{array}{l}\text { Corpus callosum } \\
\text { segment }\end{array}$ & OBPP & $\begin{array}{l}\text { Healthy } \\
\text { control }\end{array}$ & $P$-value \\
\hline Prefrontal & $56.7 \pm 16.49$ & $78.7 \pm 17.23$ & .019 \\
\hline Premotor/SMA & $102.4 \pm 24.71$ & $126.3 \pm 25.13$ & .046 \\
\hline Primary motor & $42.7 \pm 10.41$ & $52.4 \pm 10.96$ & .058 \\
\hline Primary sensory & $18.6 \pm 5.12$ & $25.7 \pm 8.21$ & .032 \\
\hline Parietal-occipital & $99.2 \pm 35.39$ & $122.3 \pm 32.47$ & .146 \\
\hline
\end{tabular}

Results of t-test.

the importance of neural plasticity in adults following successful brachial plexus neurotization. Aberrant neuroplasticity is also responsible for phantom limb pain and post-brachial plexus avulsion neuropathic pain. ${ }^{39,40}$ In fact, cortical plasticity helps in isolating respiration-synkinetic movements of the neurotized limb after an intercostal-musculocutaneous nerve transfer. With continued training, the subjects are able to isolate chest movements and limb movements. ${ }^{20}$ In another work by our group (as yet unpublished), we have discussed the role of aberrant cortical reorganization in the pathogenesis of post-brachial plexus avulsion neuropathic pain, and how DREZotomy causes the expanded neighboring sensory territory in the brain to recede, thus alleviating the pain perception. Topographical changes have been found to occur following injury per se and following recovery-spontaneous or postsurgical. After a successful intercostal-musculocutaneous neuro- tization, there is initially a respiration-synkinetic movement, but with continued training, these patients were able to isolate these movements - an effect which is attributed to neuroplasticity. ${ }^{20}$

Our results highlight the lacunae we have in understanding the cortical plasticity and outcomes in OBPP. Although the reduction in activation of sensorimotor network on the side contralateral to injured limb is expected, we have also found decreased activation and shrunken area of activation in the internal control, as compared to that in healthy controls. It is a common understanding that the right and left hemispheres are inherently different in normal individuals. ${ }^{41}$ Lateralization has been suggested to exist for visual, default mode, salience, and language networks in rsfMRI, ${ }^{42,43}$ but it is unclear that there is differential activation of sensorimotor network on both sides. Newborns are believed to be ambidextrous, or probably with vacillating hand preference, and handedness is not clearly established at least until $1 \mathrm{yr}$ of age. ${ }^{44-46} \mathrm{We}$ are not able to determine the equipotentiality of the hemispheres; thus, the assumption of internal control should be understood with this liability.

\section{Corpus Callosum Volume Loss in OBPP}

This study showed corpus callosum volume loss in subjects with OBPP. We suggest that this may be due to retrograde transneuronal degeneration. Chimelli et $\mathrm{al}^{47}$ had performed sciatic nerve section in new-born rats. They demonstrated reduced number of both myelinated and unmyelinated axons in the ipsilateral corticospinal tract fibers in the spinal cord, and also decreased number of horseradish peroxidase-labeled cells in the contralateral sensorimotor cortex. This was the first description 


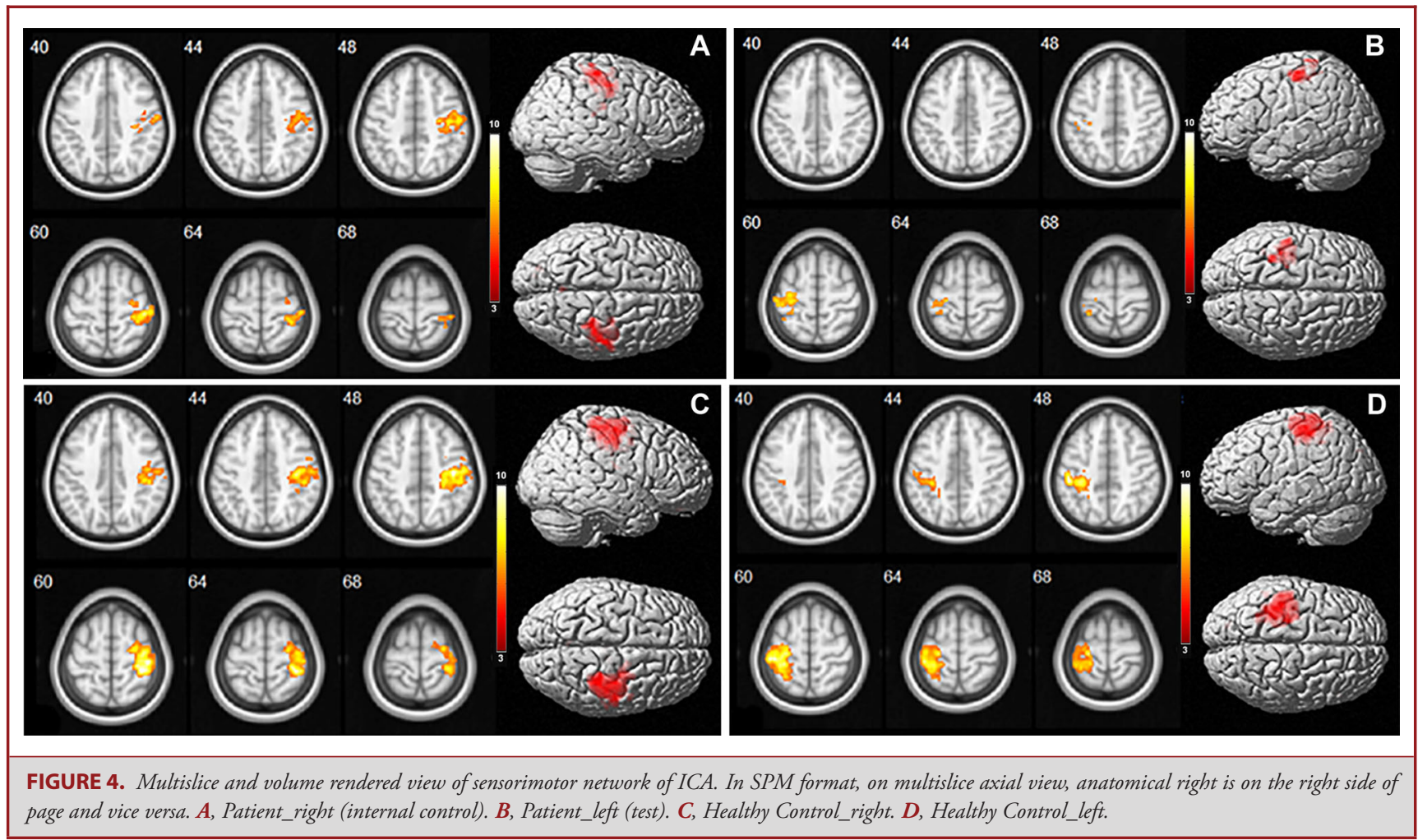

of secondary transneuronal degeneration in the brain following a peripheral nerve injury. Since that study was also performed in new-born rats, it is comparable to OBBP.

In this study, significant loss of corpus callosum volume occurred in segments I, II, and IV, which correspond to fibers from prefrontal, premotor/supplementary motor, and primary sensory areas, respectively. This correlates well with the clinical picture. In the absence of sensorimotor inputs from the injured brachial plexus, there is consequent degeneration of fibers from the motor association areas, which are responsible for motor task planning and execution. Surprisingly, the volume loss in segment III (primary motor area) is not significant. It is possible that attempts at cortical plasticity in the primary motor area result in restoration of some volume. Diffusion tensor imaging (DTI) and white matter tractography ${ }^{15,48}$ would probably give better answers regarding the functional integrity/repair of the white matter. We are looking into this aspect as well.

\section{Corroborative Evidence for Role of Corpus Callosum in Interhemispheric Coactivation}

An interesting observation is the decreased activity in the other half of the brain as well, in comparison with healthy controls. The detection of corpus callosum atrophy in this context points to its possible causative association with disruption of interhemispheric coactivation. Although some investigators have shown that corpus callosum is less important for sensorimotor interhemispheric connections, it may still be a possible contributing factor. ${ }^{9,10,49}$ Further studies are needed to improve our understanding. In this regard, it may be worth studying other restingstate networks of the brain as well.

\section{Limitations}

Although the corpus callosum is a good marker for white matter volumetry, DTI and white matter tractography would give more insights into the integrity of white matter.

Also, in view of the findings suggestive of a global insult to the developing brain, a functional connectivity MRI would further enrich our understanding. We are studying this aspect and hope to contribute to further our understanding of pediatric brain.

\section{CONCLUSION}

This study has helped to gain new insights on the developing brain's response to insults. Corpus callosum atrophy in OBPP suggests existence of more profound central effects of a peripheral nerve injury on the brain than commonly thought. What may seem to be a peripheral nerve injury may well be translated into higher cortical as well as subcortical consequences like corpus callosum volume loss and subsequent disruption of bilateral connectivity in the brain. It needs to be further established whether this is a function of the location of the injuryperipheral—or timing of the injury — neonatal immature brainor both. 


\section{Disclosures}

This study is based on the thesis of Dr Kishore Kislay and part of the research was funded by Indian Council of Medical Research (ICMR) Thesis grant (3/2/Nov-2014/PG-Thesis-HRD-17). The authors have no personal, financial, or institutional interest in any of the drugs, materials, or devices described in this article.

\section{REFERENCES}

1. Dobbing J, Sands J. Quantitative growth and development of human brain. Arch Dis Child 1973;48(10):757-767.

2. Brody BA, Kinney HC, Kloman AS, Gilles FH. Sequence of central nervous system myelination in human infancy. I. An autopsy study of myelination. J Neuropathol Exp Neurol 1987;46(3):283-301.

3. Dietrich R, Bradley W, Zaragoza E, et al. MR evaluation of early myelination patterns in normal and developmentally delayed infants. Am J Roentgenol 1988;150(4):889-896.

4. Cowan WM. Anterograde and retrograde transneuronal degeneration in the central and peripheral nervous system. In: Nauta WJH, Ebbesson SOE, eds. Contemporary Research Methods in Neuroanatomy. Springer: Berlin Heidelberg; 1970:217-251. Available at: http://link.springer.com/chapter/10. 1007/978-3-642-85986-1_11. Accessed November 17, 2016.

5. Beatty R, Sadun A, Smith L, Vonsattel J, Richardson E. Direct demonstration of transsynaptic degeneration in the human visual system: a comparison of retrograde and anterograde changes. J Neurol Neurosurg Psychiatry 1982;45(2): 143-146.

6. Bridge H, Plant GT. Conclusive evidence for human transneuronal retrograde degeneration in the visual system J Clin Exp Ophthalmol 2012;S3:003.

7. Benavidez DA, Fletcher JM, Hannay HJ, et al. Corpus callosum damage and interhemispheric transfer of information following closed head injury in children. Cortex 1999;35(3):315-336.

8. Lou L, Shou T, Li Z, Li W, Gu Y. Transhemispheric functional reorganization of the motor cortex induced by the peripheral contralateral nerve transfer to the injured arm. Neuroscience 2006;138(4):1225-1231.

9. Johnston JM, Vaishnavi SN, Smyth MD, et al. Loss of resting interhemispheric functional connectivity after complete section of the corpus callosum $J$ Neurosci 2008;28(25):6453-6458.

10. Hua X, Li Z, Xu W, Zheng $\mathrm{M}, \mathrm{Xu}$ J, Gu Y. Interhemispheric functional reorganization after cross nerve transfer: via cortical or subcortical connectivity? Brain Res 2012;1471:93-101.

11. Ewing-Cobbs L, Prasad MR, Hasan KM. Developmental plasticity and reorganization of function following early diffuse brain injury. In: Nelson CA, Luciana M, eds. Handbook of Developmental Cognitive Neuroscience. London, UK: MIT Press; 2008:399-413.

12. Levin HS, Benavidez DA, Verger-Maestre K, et al. Reduction of corpus callosum growth after severe traumatic brain injury in children. Neurology 2000;54(3):647647.

13. Bigler ED. Quantitative magnetic resonance imaging in traumatic brain injury. J Head Trauma Rehabil 2001;16(2):117-134.

14. Beauchamp $\mathrm{MH}$, Anderson VA, Catroppa $\mathrm{C}$, et al. Implications of reduced callosal area for social skills after severe traumatic brain injury in children. J Neurotrauma 2009;26(10):1645-1654.

15. Wu TC, Wilde EA, Bigler ED. Longitudinal changes in the corpus callosum following pediatric traumatic brain injury. Dev Neurosci 2010;32(5-6):361-373.

16. Rosazza C, Minati L. Resting-state brain networks: literature review and clinical applications. Neurol Sci 2011;32(5):773-785.

17. Lee MH, Smyser CD, Shimony JS. Resting-state fMRI: a review of methods and clinical applications Am J Neuroradiol 2013;34(10):1866-1872.

18. Lang S, Duncan N, Northoff G. Resting-state functional magnetic resonance imaging. Neurosurgery 2014;74(5):453-464; discussion 464-465.

19. Navarro X, Viv? M, Valero-Cabr? A. Neural plasticity after peripheral nerve injury and regeneration. Prog Neurobiol 2007;82(4):163-201.

20. Sokki AM, Bhat DI, Indira Devi B. Cortical reorganization following neurotization. Neurosurgery 2012;70(5):1305-1311; discussion 1311.

21. Dimou S, Biggs M, Tonkin M, Hickie IB, Lagopoulos J. Motor cortex neuroplasticity following brachial plexus transfer. Front Hum Neurosci 2013;7:500.
22. Mohanty CB, Bhat D, Indira Devi B. Role of central plasticity in the outcome of peripheral nerve regeneration. Neurosurgery 2015;77(3):418-423.

23. Hofer S, Frahm J. Topography of the human corpus callosum revisitedcomprehensive fiber tractography using diffusion tensor magnetic resonance imaging. NeuroImage 2006;32(3):989-994.

24. Herron TJ, Kang X, Woods DL. Automated measurement of the human corpus callosum using MRI. Front Neuroinform 2012;6:25.

25. Adamson C, Beare R, Walterfang M, Seal M. Software pipeline for midsagittal corpus callosum thickness profile processing. Neuroinformatics 2014;12(4):595614.

26. Smith SM, Jenkinson M, Woolrich MW, et al. Advances in functional and structural MR image analysis and implementation as FSL NeuroImage 2004;23(suppl 1):S208-S219.

27. Jenkinson M, Bannister P, Brady M, Smith S. Improved optimization for the robust and accurate linear registration and motion correction of brain images. NeuroImage 2002;17(2):825-841.

28. Smith SM. Fast robust automated brain extraction. Hum Brain Mapp 2002;17(3):143-155

29. Beckmann CF, Smith SM. Tensorial extensions of independent component analysis for multisubject FMRI analysis. NeuroImage 2005;25(1):294-311.

30. Beckmann CF, Deluca M, Devlin JT, Smith SM. Investigations into restingstate connectivity using independent component analysis. Philos Trans Roy Soc B Biol Sci 2005;360(1457):1001-1013.

31. Klein A, Andersson J, Ardekani BA, et al. Evaluation of 14 nonlinear deformation algorithms applied to human brain MRI registration. NeuroImage 2009;46(3):786 802.

32. Beckmann CF, Mackay, CE, Filippini N, Smith, SM. Group comparison of resting-state FMRI data using multi-subject ICA and dual regression. NeuroImage 2009; $47:$ : 148

33. Filippini N, Macintosh BJ, Hough MG, et al. Distinct patterns of brain activity in young carriers of the APOE- 4 allele. Proc Natl Acad Sci USA 2009;106(17):72097214.

34. Qiu T-M, Chen L, Mao Y, et al. Sensorimotor cortical changes assessed with resting-state fMRI following total brachial plexus root avulsion. J Neurol Neurosurg Psychiatry 2014;85(1):99-105.

35. Zhang D, Johnston JM, Fox MD Preoperative sensorimotor mapping in brain tumor patients using spontaneous fluctuations in neuronal activity imaged with functional magnetic resonance imaging: initial experience. Neurosurgery 2009;65(6 suppl):226-236.

36. Kannurpatti SS, Rypma B, Biswal BB. Prediction of task-related BOLD fMRI with amplitude signatures of resting-state fMRI. Front Syst Neurosci 2012; $6: 7$

37. Rosazza C, Aquino D, D'Incerti L, et al. Preoperative mapping of the sensorimotor cortex: comparative assessment of task-based and resting-state fMRI. PLoS ONE 2014;9(6):e98860.

38. Eysel UT. Adult cortical plasticity. In: Squire LR, ed. Encyclopedia of Neuroscience Oxford: Academic Press; 2009:141-147. Available at: http:/www.sciencedirect. com/science/article/pii/B9780080450469002011. Accessed May 14, 2015.

39. Simon NG, Franz CK, Gupta N, Alden T, Kliot M. Central adaptation following brachial plexus injury World Neurosurg 2016;85:325-332.

40. Lotze M, Grodd W, Birbaumer N, Erb M, Huse E, Flor H. Does use of a myoelectric prosthesis prevent cortical reorganization and phantom limb pain? Nat Neurosci 1999;2(6):501-502.

41. Feng J, Liu $\mathrm{H}, \mathrm{Xu} J, \mathrm{Gu}$ Y, Shen Y. Differences in brain adaptive functional reorganization in right and left total brachial plexus injury patients. World Neurosurg 2015;84(3):702-708

42. Liu H, Stufflebeam SM, Sepulcre J, Hedden T, Buckner RL. Evidence from intrinsic activity that asymmetry of the human brain is controlled by multiple factors. Proc Natl Acad Sci USA 2009;106(48):20499-20503.

43. Nielsen JA, Zielinski BA, Ferguson MA, Lainhart JE, Anderson JS, He Y. An evaluation of the left-brain vs. right-brain hypothesis with resting state functional connectivity magnetic resonance imaging. PLoS ONE 2013;8(8):e71275.

44. Gesell A, Ames LB. The development of handedness $J$ Genet Psychol $1947 ; 70(2): 155-175$

45. Bates E, O'connell B, Vaid J, Sledge P, Oakes L. Language and hand preference in early development. Dev Neuropsychol 1986;2(1):1-15.

46. Nespoulous JL, Perron P, Lecours AR. The Biological Foundations of Gesture: Motor and Semiotic Aspects. Hove (UK): Psychology Press, 2014 
47. Chimelli L, Scaravilli F. Secondary transenuronal degeneration: cortical changes induced by peripheral nerve section in neonatal rats. Neurosci Lett 1985;57(1):5763.

48. Bendlin BB, Ries ML, Lazar M, et al. Longitudinal changes in patients with traumatic brain injury assessed with diffusion-tensor and volumetric imaging. NeuroImage 2008;42(2):503-514.

49. Pawela CP, Biswal BB, Hudetz AG, et al. Interhemispheric neuroplasticity following limb deafferentation detected by resting-state functional connectivity magnetic resonance imaging ( $\mathrm{fcMRI})$ and functional magnetic resonance imaging (fMRI). NeuroImage 2010;49(3):2467-2478.

\section{COMMENT}

he effects of neonatal brachial plexus injury (NBPP) on the developing brain are still largely unknown. This very interesting paper uses fMRI to describe the effects of NBPP on corpus callosum volumetry, especially in the motor association areas. Unfortunately, due to the small number of patients and healthy controls that were included in the study, it was not possible to determine any statistical differences between operated and non-operated individuals. Nevertheless, this paper gives some minimal clues for explanation of several facts that are observed after a NBPP surgical reconstruction: patients showing adequate reinnervation of muscles that are not functionally used, bilateral activation of the cortex when performing a single-limb task, changes in language dominancy from the affected to the healthy hemisphere, or patients under 15 years old having a contralateral $\mathrm{C} 7$ transfer achieving independent control of both upper limbs. Future studies with a bigger number of patients will be more than welcomed in the future in order to further clarify these items and many others.
Mariano Socolovsky Buenos Aires, Argentina 